\title{
FEATURE
}

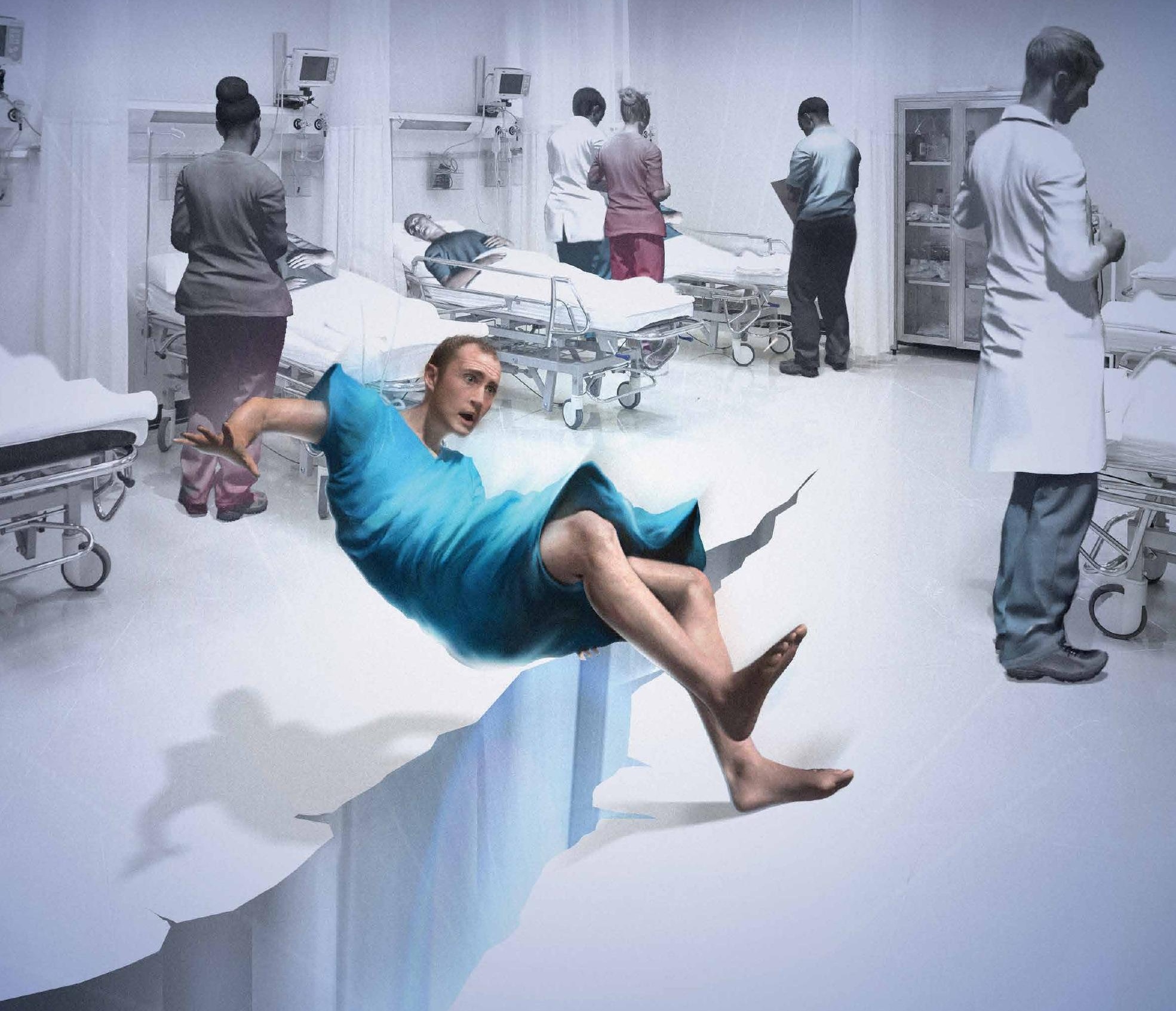




\section{Patient Safety in the Emergency Department}

Brenna M. Farmer, MD

\section{The ED is inherently a high-risk setting for errors that can result in patient harm. This article outlines strategies for improving medication safety, transitions of care, health information technology, and other factors.}

Patient safety has received increased attention since the late 1990s. In 1999, The Institute of Medicine published "To Err is Human: Building a Safer Health System," followed by "Crossing the Quality Chasm: A New Health System for the 21st Century" in 2001 to document patient-safety issues and recommend improvements in medical care to reduce errors. These reports and other patient-safety studies, however, likely underestimate the extent of medical errors and preventable harm. After these reports appeared, many specialties began to seriously evaluate their own safety issues.

Among the specialties, emergency medicine (EM) identified several problem areas and attempted to determine the epidemiology of errors. One study of 62 urban EDs found that at least $7 \%$ of patients who presented for myocardial infarctions (MIs), asthma exacerbations, or joint dislocations requiring reduction with procedural sedation experienced an actual or near-miss adverse event. ${ }^{3}$ Another study showed that up to $12 \%$ of all return visits to the ED within 7 days were related to adverse events. ${ }^{4}$
The ED setting itself undoubtedly contributes significantly to the risk of harm. This article illustrates and discusses ED patient-safety issues, and offers some recommendations for improvement in care and prevention of harm.

\section{The ED Setting}

The ED is unlike any other area of the hospital or health-care setting. Patients seek care for both primary care and urgent care complaints at any time of the day or night, on any day of the week, when no other source of care is available. Emergency physicians (EPs) are required to care for multiple patients of different ages while prioritizing care of the critically ill who have MI, stroke, sepsis, respiratory distress, or multisystem trauma. For many ED patients, diagnosis and treatment can be complex.

The ED setting is fast-paced and requires quick thinking, a broad depth of knowledge about many medical conditions, and a broad range of skills to perform emergent and lifesaving procedures. Often, patients are presenting to a hospital ED for the first time, with incomplete medical records. They may

Dr Farmer is an assistant professor of clinical medicine, Weill Cornell Medical College, New York, New York; and an emergency physician and toxicologist, NY Presbyterian Hospital, New York.

DOI: 10.12788/emed.2016.0052 
In general, medication errors are 13.5 times less likely to occur when a pharmacist is on duty in the ED. not know their medical conditions or medications, or be in a position to communicate this information. Any of these situations alone can lead to an adverse event; in combination, they can significantly increase the risk for harm. In addition, ED overcrowding due to limited availability of inpatient hospital beds may consume resources and staffing needed to care for active ED patients and new patients coming through the door.

Safety factors in the ED can be categorized as those related to patients, providers, or the environment/systems (Table 1)..$^{5-7}$ When a large academic urban ED studied its errors, two-thirds were attributed to systems issues. ${ }^{5}$

\section{Culture of Safety}

Developing and maintaining a "culture of safety" is a commitment to minimize adverse events when performing high-risk jobs that can result in harm. ${ }^{8}$ This concept originated in other industries such as the airline and nuclear energy industries. Organizations and companies are considered high-reliability organizations (HROs) when they are dedicated to preventing harm at all staff levels-from the frontline to the corporate level. These HROs promote the reporting of errors and "near misses" without fear of blame or loss of employment. ${ }^{8}$ In the ED, a culture of safety encourages teamwork, event reporting, communication openness, transparency with feedback and learning from errors, and administrator collaboration for safety. ${ }^{9}$

In EDs with a strong safety culture, near misses are more likely to be intercepted to reduce patient harm. ${ }^{3}$ Teamwork training improves communication and reduces errors. ${ }^{10}$ One such program, Team Strategies and Tools to Enhance Performance and Patient Safety (TeamSTEPPS), was developed by a joint effort of the US Department of Defense and Agency for Healthcare Research and Quality to promote interprofessional communication between all providers in the hospital. This program provides many tools, including one to obtain attention in difficult situations and one to escalate concerns to focus on an important safety issue. ${ }^{11}$ One ED's experience with TeamSTEPPS led it to identify specific steps to ensure continued success after the initial start. To maintain the high level of teamwork and successful communication, this ED recognized a need for continued champions at all staff levels and all new staff members were required to go through the training. ${ }^{12}$

Another important aspect of a strong safety culture is creating an environment that promotes reporting of adverse events and near misses. The culture should allow a person involved in an adverse event to feel comfortable reporting such events. In one study of 522 "unintended events" at 10 EDs in the Netherlands, nurses reported $85 \%$ of events, and resident physicians reported $13 \%$ of events. Approximately $83 \%$ of reports were filed by a person involved in the event. ${ }^{13}$ This study highlights EDs that foster a "no blame" environment, where staff members feel comfortable admitting mistakes, and there is no fear of punishment or concern for job loss. When administration supports such reporting, the true safety problems in the ED are identified and can be targeted for improvement.

\section{Medication Safety}

\section{Case Scenario 1}

A 65-year-old woman presented to the ED with atrial fibrillation with a rapid ventricular rate of 165 beats/minute. Her heart rate was controlled with intravenous (IV) diltiazem, and a heparin infusion was ordered based on her estimated weight of $150 \mathrm{lb}$. As the pharmacist prepared the infusion, she rechecked the patient's weight and discovered that the heparin order had been placed using pounds instead of kilograms. The pharmacist discussed the order with the physician, and the order was changed to avoid a double-dosing error.

\section{Discussion}

Many medications are required to treat critical illnesses and complex medical con- 
Table 1. Safety Factors in the Emergency Department ${ }^{5-7}$

\begin{tabular}{lll}
\hline Patient-Related & Provider-Related & Environment/System-Related \\
\hline Acuity and emergent conditions & Experience & Inadequate staffing \\
Age extremes & Fatigue, shift work & Inexperienced staff \\
Communication barriers & Cognitive errors & Teamwork and communication problems \\
Vague or atypical complaints & Missed test results & Overcrowding \\
Undifferentiated presentations & Procedural errors & Boarding of admitted patients \\
Mental status changes & Policy deviation & Lack of equipment or equipment failures \\
Cognitive impairment & Transitions of care & Reliability of consultation services \\
Complex medical conditions & & Inadequate consultation services \\
Lack of knowledge of medical & & Lack of complete medical records \\
problems and medications & & Difficulty using electronic health record \\
& & or order entry system \\
\hline
\end{tabular}

ditions; such polypharmacy is further complicated by the sheer volume of patients seen in the ED. The wide range of medications used in the ED and the different doses appropriate for age, gender, and body weight can lead to patient harm when the prescriber is confused. In addition, many medications can be administered via multiple routes, including IV, intramuscular, subcutaneous, or oral. In situations where a critically ill patient is close to death, verbal orders are often used and then followed by computer orders when the physician is able to leave the bedside. Clinicians may be simultaneously treating multiple patients with similar conditions or with similar names. In addition, due to the acuity of patient complaints, "high-alert" medications are often used in the ED, ${ }^{14}$ such as paralytics, opioids, anticoagulants, antithrombotics, insulins, sedatives, and vasopressors. ${ }^{15}$ Considering all of these factors, it is not surprising that up to $60 \%$ of ED patients experienced medication errors in one study. ${ }^{16}$ Fortunately, most of these errors do not result in immediate patient harm, but have the potential to lead to harm. ${ }^{17}$

The addition of a pharmacist to the ED 24 hours a day, 7 days a week can greatly improve medication safety. Emergency depart- ment pharmacists are available for immediate bedside consultation or discussion of a medication order, and can intercept prescribing errors in the ordering system before they are administered and before they result in patient harm. ${ }^{18}$ In general, medication errors are 13.5 times less likely to occur when a pharmacist is on duty in the ED. ${ }^{19}$ Pharmacists can recommend appropriate antibiotic dosing, ${ }^{20}$ as well as aid in the timely administration of medications for such emergent conditions and procedures as stroke, MI, trauma, and rapid-sequence intubation. In our ED, the pharmacists also ensure that look-alike/sound-alike (LASA) medications are not confused. Importantly, in overcrowded EDs, the pharmacist reviews medication orders for all inpatients boarding in the ED and ensures that the nurses obtain the appropriate medications from the automated dispensing cabinets. In some instances, neither the EP nor the ED nurses may be familiar with proper doses and scheduling of medications typically used only in the inpatient service.

Pharmacists can prevent errors with formulation confusion, LASA confusion, weight-based dose errors, and dosing frequency errors. They also can ensure that the most up-to-date evidence is used to 
support a medication ordered, ensuring best practices and adherence to hospital policies. Table $2^{14}$ summarizes additional information on best practices for medication safety in the ED.

\section{Discharge Process}

\section{Case Scenario 2}

A 55-year-old man on warfarin presented to the ED with cough, dyspnea, and fever. His chest X-ray revealed right lower lobe pneumonia. He was prescribed levofloxacin and discharged home. His discharge instructions included a discussion of pneumonia, fever control, and the importance of taking his antibiotic appropriately, but he was not told to have his international normalized ratio (INR) checked regularly while taking levofloxacin. When the patient returned to the ED 5 days later because of rectal bleeding, his INR was elevated to 6 (normal range in a patient taking warfarin is 2.0-3.0).

\section{Discussion}

When patients who do not require admission to the hospital are discharged home, they need instructions to ensure that they fully understand the nature of their problem and what they need to do to get better. For the provider, the discharge process

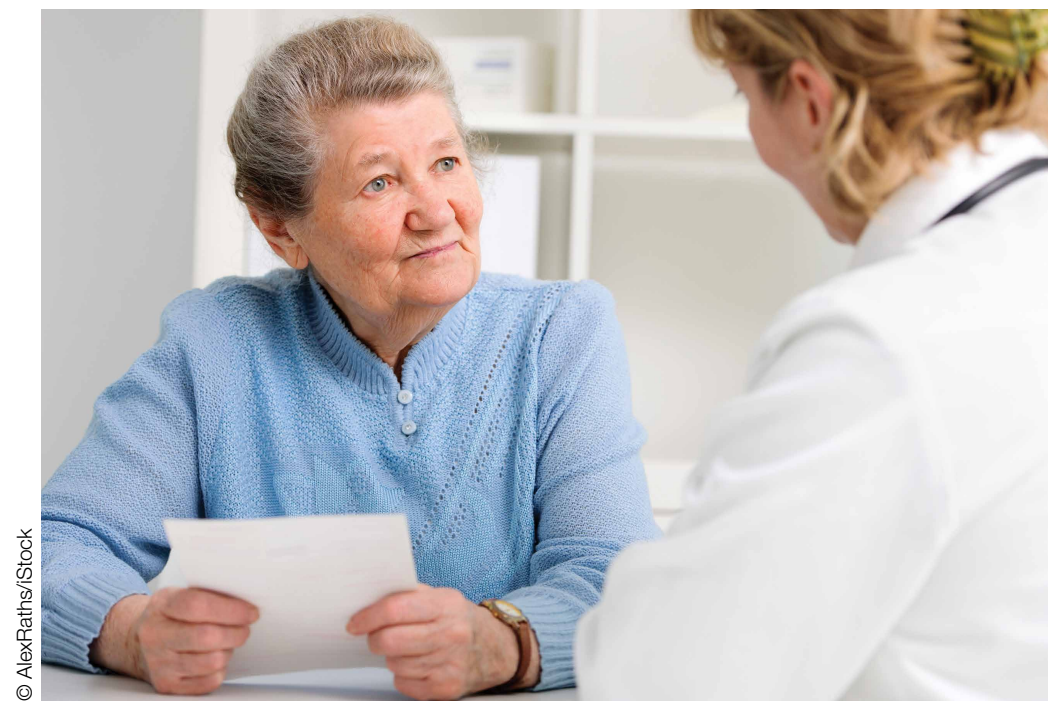

must include three tasks: communicating crucial information (diagnosis and return precautions), verifying the patient's comprehension of the information presented, and addressing and correcting specific concerns and misunderstandings. ${ }^{21}$ The encounter must be standardized but also be flexible enough to ensure patient understanding across a wide range of health care literacy and cultural backgrounds. ${ }^{21} \mathrm{~Pa}-$ tients frequently are not given appropriate verbal and written instructions, and if they do not understand their diagnosis, they may not follow up when necessary; may not realize that they need to take specific medications; or may not take their newly prescribed medications as intended.

In an evaluation of written discharge instructions, only $76 \%$ included a diagnosis or an explanation of the patient's symptoms, and only $34 \%$ provided instructions on when and how to return. ${ }^{22}$ Another study of the discharge process showed that the average verbal discharge exchange lasted only 76 seconds and that $65 \%$ of instructions were not complete. Patients were often not given a diagnosis, an explanation of their prescriptions, or proper return precautions. ${ }^{23}$ Deficits in the discharge process places patients at risk for medical and medication errors.

The discharge exchange must provide information on the diagnosis, what was done in the ED, and what needs to happen next. This must be done both verbally and in writing, in the patient's native language, and at his or her health-literacy level. There should be time for the patients and those accompanying them and who are also responsible for their health to ask questions to ensure that everyone understands what has taken place and what must be done after leaving the ED. Patients should be given information on all prescription and over-the-counter medications they are instructed to take, as well as any changes to their previously prescribed medications.

Patients should be told specifically with whom to follow up and within what time 
Table 2. Best Medication Safety Practices in the Emergency Department ${ }^{14}$

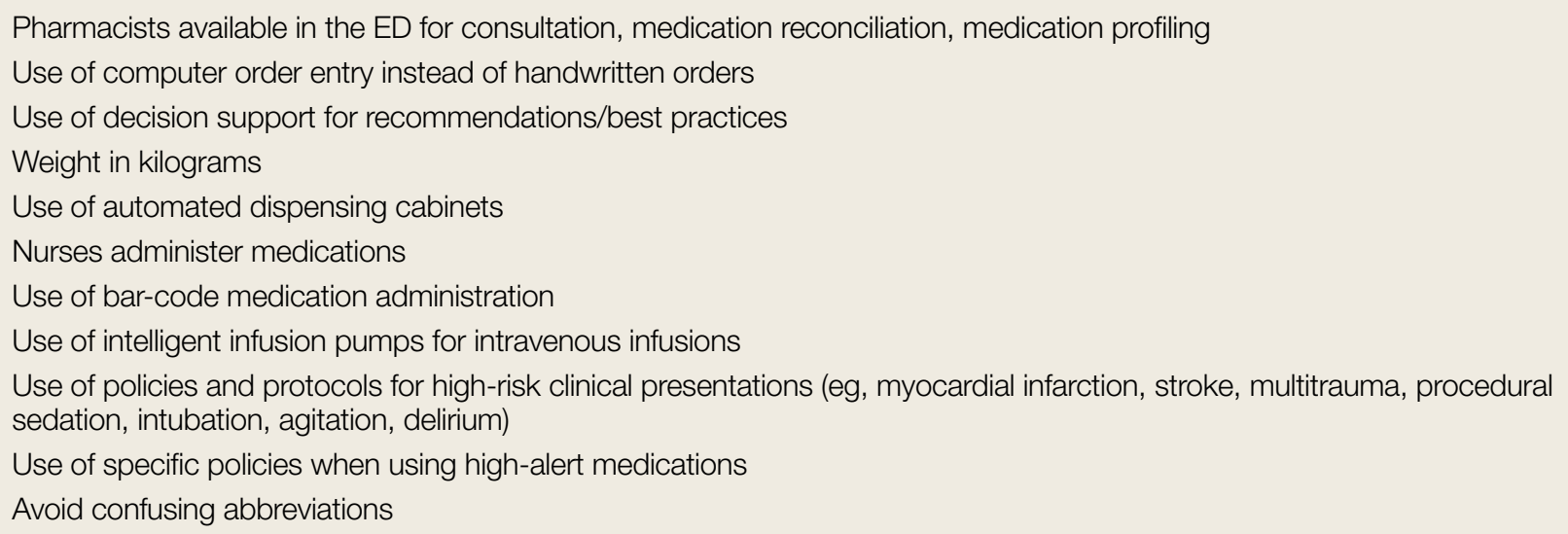

frame. If possible, the exact time and location of a follow-up appointment should be provided. For patients with lower health literacy and less understanding of the health-care system, a process should be in place to help them navigate and ensure they get to necessary appointments. ${ }^{21}$

\section{Handoffs and Transitions of Care Case Scenario 3}

A 70-year-old man with hypertension and hyperlipidemia had an episode of chest pain and was evaluated in the ED for possible myocardial ischemia. His initial electrocardiogram was interpreted as nonischemic and his troponin level was below detection 30 minutes after the episode. As the initial provider was leaving the ED, he endorsed the patient to the oncoming EP, with instructions to follow up on the chest $\mathrm{X}$-ray interpretation. The initial provider, however, did not tell the oncoming EP to check the results of a repeat troponin determination. The patient was discharged home after the second troponin test had been sent to the laboratory, but before the results had been checked.

\section{Discussion}

Emergency department patients still under evaluation or in the process of be- ing admitted to the inpatient hospital are "handed off" to the next shift of providers. Handoffs, or transitions of care, place patients at high risk for adverse events or bad outcomes. Important information can be lost whenever care is transferred to another provider. For example, there can be a lack of communication about pending tests that require follow-up, the need for further testing, or contingency planning for any problems that may arise. Loss of information and lack of follow-up can lead to diagnostic error and improper disposition.

According to the Joint Commission and a 2006 National Patient Safety Goal, handoffs should be standardized. ${ }^{24}$ The four stages for safe ED-provider-to-ED-provider handoffs are pre-turnover, arrival of new provider, meeting of providers, and post-turnover. ${ }^{25}$ During pre-turnover, the initial provider should review what has happened in the patient's care and the next steps needed to finalize patient disposition. The arrival of the new provider signals the start of a new shift. During the meeting with the new provider, important information should be verbally transmitted to the oncoming provider. ${ }^{25}$ This meeting needs to be standardized to include a patient summary, tasks and tests to follow up, and contingency planning. Many tools 
Table 3. Transitions of Care ${ }^{25,26}$

\begin{tabular}{lcc}
\hline Safety Improvement & $\begin{array}{c}\text { ED Provider } \\
\text { to ED Provider }\end{array}$ & $\begin{array}{c}\text { ED Provider } \\
\text { to Inpatient Provider }\end{array}$ \\
\hline Standardized verbal handoff & $X$ & $\times$ \\
Standardized written handoff & $X$ & $X$ \\
Limit the number of patient handoffs & $X$ & $X$ \\
Handoffs should include: & & \\
Patient summary & $X$ & $X$ \\
To-do list & $X$ & $X$ \\
Time for new provider to ask questions & $X$ & $X$ \\
Closed loop communication between providers & $X$ & $X$ \\
\hline
\end{tabular}

can aid in transitions of care, including verbal mnemonics, tools to integrate with the medical record, and tools to develop a complete process for transition of care. Post-turnover is completed by the oncoming provider as he or she finishes any tasks related to the patient's care to ensure the treatment plan is completed..$^{25}$

There are many ways to improve the safety of handoffs. First, the number of handoffs should be limited. Having more patients dispositioned by the provider who initiated their care reduces the risk of an adverse event. This can be accomplished by having overlapping shifts to allow outgoing providers time to complete care for their patients. During handoffs, interruptions and distractions should be limited to give the off-going provider appropriate time to present a succinct but complete overview of the patient's care and communicate all outstanding tasks as "to-do" or "action lists," with contingency planning for any changes in the patient's status, test results, etc. There should be time for the oncoming provider to ask questions to ensure he or she is clear about the next steps. ${ }^{25}$ At the end of the transition, there should be some signal that the patient's care is passed on to the oncoming provider and the outgoing physician should leave the patient-care area to finish documentation.

Many ED patients will need transition from "ED patient" to "admitted patient"ie, admission to the hospital and transfer of care to an inpatient service provider. Studies on transitions of care from the ED to an inpatient medical service have found multiple barriers to a seamless transition of care. These include communication failures; information technology failures; inability of inpatient providers to review vital signs, laboratory values, and medications given; a change of the inpatient team to whom the patient was assigned; and patient transfers to areas remote from the ED and/or inpatient floors, such as to a dialysis unit. In one survey, $29 \%$ of respondents reported that a patient of theirs had experienced an adverse event or near misses due to a poor handoff between the ED and medical service. ${ }^{26}$ Just as there needs to be a standardized process for EDprovider-to-ED-provider handoffs, there also should be a standardized process for ED-to-inpatient or -outpatient service provider handoffs. There should be verbal and possibly written transmission of vital information, with patient summaries, "to-do" lists of follow-ups, situational knowledge with contingency planning, and time for questions (Table 3). ${ }^{25,26}$ The Joint Commission's Transitions of Care Portal (https://www.jointcommission.org /toc.aspx) offers tools to help facilities formalize this process. 


\section{Health Information Technology}

\section{Case Scenario 4}

An EM intern was instructed to order a dose of morphine for a patient with a fractured hip. The intern used electronic ordering. Afterward, the nurse caring for the patient asked the attending EP if she really wanted to order patient-controlled morphine analgesia for the patient. Upon reviewing the order, the attending discovered the intern had selected the first morphine on the drop-down list instead of scrolling down to find the range of individual doses available.

\section{Discussion}

The use of electronic health records (EHRs) and health information technology (HIT) systems has both improved patient care and introduced new errors. Physician handwriting may no longer be a problem, but some hospitals use several types of EHRs simultaneously, with different systems for inpatients, outpatients, and EDs. In these settings, there may not be a seamless system to allow for review of inpatient, outpatient, and ED records. Additional concerns include communication failure, misidentification of patient orders, poor data display, and "alert fatigue." ${ }^{27}$ Communication failures include the lack of bedside or face-to-face discussion among care providers. Physicians may enter orders at a computer away from the nursing station and never directly inform the nurse about the plan for the patient.

Incorrect patient orders are usually selfexplanatory. Other errors include choosing the wrong LASA medication from a dropdown list or ordering imaging studies for the wrong side of the patient's body. Poor data display may not alert providers of two or more patients with the same last name or allow vital signs to be displayed in a meaningful way. Other data-display problems include the inability to distinguish abnormal results from normal results because the system uses the same display color for both. Conversely, alert fatigue

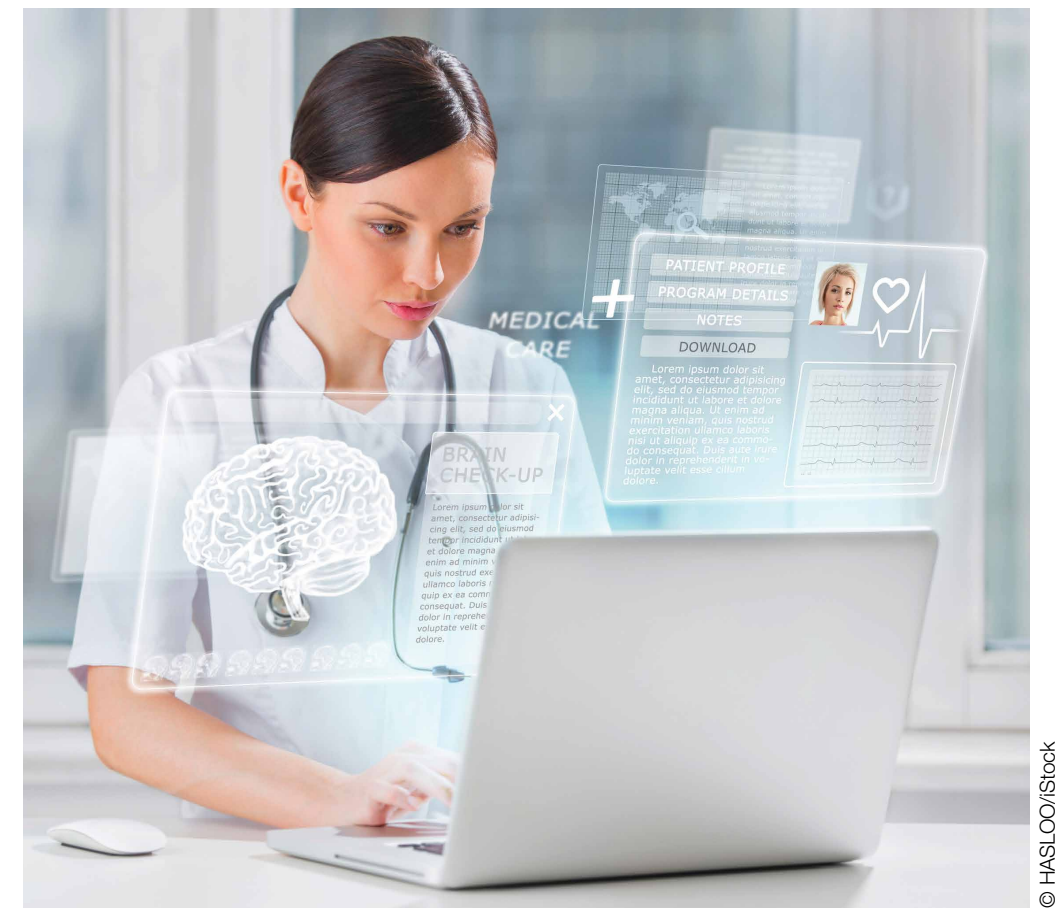

occurs when too many warning messages appear while providers are trying to enter orders for patient care. These warnings can range from important messages such as allergy identification or severe drug interactions to noncritical alerts about the cost of a test.

Recommendations to improve patient safety with the use of EHRs or HIT systems involve having a frontline staff champion to identify areas for performance improvement and having a review process to identify and examine safety issues with these technologies. A multidisciplinary group, including frontline staff, can usually develop effective solutions to these safety issues. $^{27}$

\section{Conclusion}

The ED is a high-risk setting for errors because it features high-acuity patients, patients of widely divergent ages, the frequent need to use high-alert medications, the need to simultaneously care for multiple patients, many interruptions and distractions, and the lack of an established 
relationship with patients. This environment can lead to communication failures in handoffs and transitions of care, medication errors, and poor follow-up due to poor discharge processes. Additional difficulties arise when HIT systems, such as EHRs, are not set up to ensure the success of frontline staff caring for ill patients. The ED can become a much safer place by establishing strategies such as those outlined in this article to reduce error in all of these areas.

\section{References}

1. Institute of Medicine. To Err is Human: building a Safer Health System. LT Kohn, JM Corrigan, MS Donaldson, eds. Washington, DC: National Academy Press, 1999.

2. Institute of Medicine. Crossing the Quality Chasm: a New Health System for the 21st Century. Washington, DC: National Academy Press, 2001.

3. Camargo CA Jr, Tsai CL, Sullivan AF, et al. Safety climate and medical errors in 62 US emergency departments. Ann Emerg Med. 2012;60(5):555-563.e20.

4. Calder L, Pozgay A, Riff S, et al. Adverse events in patients with return emergency department visits. BMJ Qual Saf. 2015;24(2):142-148.

5. Jepson ZK, Darling CE, Kotkowski KA, et al. Emergency department patient safety incident characterization: an observational analysis of the findings of a standardized peer review process. BMC Emerg Med. 2014:14:20.

6. Ramlakhan S, Qayyum H, Burke D, Brown R. The safety of emergency medicine. Emerg Med J. 2016;33(4):293-299.

7. Sklar DP, Crandall C. What do we know about emergency department safety? Perspectives on Safety. Patient Safety Network. https://psnet.ahrq. gov/perspectives/perspective/88/what-do-we-knowabout-emergency-department-safety. Published June 2010. Accessed June 30, 2016.

8. Patient Safey Network. Safety culture. https://psnet ahrq.gov/primers/primer/5/safety-culture. Updated July 2016. Accessed July 1, 2016.

9. Verbeek-VanNoord I, Wagner C, VanDyck C, Twisk JW, DeBruijne MC. Is culture associated with patient safety in the emergency department? A study of staff perspectives. Int J Qual Health Care. 2014;26(1):64-70.

10. Morey JC, Simon R, Jay GD, et al. Error reduction and performance improvement in the emergency department through formal teamwork training: evaluation results of the MedTeams project. Health Serv Res. 2002;37(6):1553-1581.

11. Agency for Healthcare Research and Quality. About TeamSTEPPS.http://www.ahrq.gov/teamstepps/ about-teamstepps/index.html. Accessed July 1, 2016.
12. Turner P. Implementation of TeamSTEPPS in the emergency department. Crit Care Nursing $Q$. 2012;35(3):208-212.

13. Smits M, Groenewegen PP, Timmermans TRM, van der Wal G, Wagner C. The nature and causes of unintended events reported at ten emergency departments. BMC Emerg Med. 2009;9:16.

14. Croskerry P, Shapiro M, Campbell S, et al. Profiles in patient safety: medication errors in the emergency department. Acad Emerg Med. 2004;11(3):289-299.

15. Institute for Safe Medicine Practices. ISMP List of High-Alert Medications in Acute Care Settings. http://www.ismp.org/Tools/highalertmedications. pdf. Updated 2014. Accessed July 15, 2016.

16. Patanwala AE, Warholak TL, Sanders AB, Erstad BL. A prospective observational study of medication errors in a tertiary care emergency department. Ann Emerg Med. 2010;55(6):522-526.

17. Patanwala AE, Hays DP, Sanders AB, Erstad BL. Severity and probability of harm of medication errors intercepted by an emergency department pharmacist. Int J Pharm Pract. 2011;19(5):358-362.

18. Patanwala AE, Sanders AB, Thomas MC, et al. A prospective, multicenter study of pharmacist activities resulting in medication error interception in the emergency department. Ann Emerg Med. 2012;59(5):369-373.

19. Ernst AA, Weiss SJ, Sullivan A 4th, et al. On-site pharmacists in the ED improve medical errors. Am J Emerg Med. 2012;30(5):717-725.

20. Dewitt KM, Weiss SJ, Rankin S, Ernst A, Sarangarm P. Impact of an emergency medicine pharmacist on antibiotic dosing adjustment. Am J Emerg Med. 2016;34(6):980-984

21. Samuels-Kalow ME, Stack AM, Porter SC. Effective discharge communication in the emergency department. Ann Emerg Med. 2012;60(2):152-159.

22. Vashi A, Rhodes KV. "Sign right here and you're good to go": a content analysis of audiotaped emergency department discharge instructions. Ann Emerg Med. 2011;57(4):315-322.e1.

23. Rhodes KV, Vieth T, He T, et al. Resuscitating the physician-patient relationship: emergency department communication in an academic medical center. Ann Emerg Med. 2004;44(3):262-267.

24. The Joint Commission. 2016 National Patient Safety Goals. http://www.jointcommission.org/PatientSafety/NationalPatientSafetyGoals/06_npsg_cah.htm. Accessed June 24, 2016.

25. Cheung DS, Kelly JJ, Beach C, et al. Improving handoffs in the emergency department. Ann Emerg Med. 2010;55(2):171-180.

26. Horowitz LI, Meredith T, Schuur JD, Shah NR, Kulkarni RG, Jeng GY. Dropping the baton: a qualitative analysis of failures during the transition from emergency department to inpatient care. Ann Emerg Med. 2009;53(6):701-710.e4.

27. Farley HL, Baumlin KM, Hamedani AG, et al. Quality and safety implications of emergency department information systems. Ann Emerg Med. 2013;62(4):399-407. 